\section{International Scientific Journal Theoretical \& Applied Science}

\author{
p-ISSN: 2308-4944 (print) e-ISSN: 2409-0085 (online) \\ Year: 2017 Issue: 09 Volume: 53
}

Published: $21.09 .2017 \quad \underline{\text { http://T-Science.org }}$

SECTION 2. Applied mathematics. Mathematical modeling.
Mohiniso Hidirova

PhD, Senior Scientist of dept. "Regulatorika"

Science and innovation center for information and communication technologies at the Tashkent University of Information Technologies named after Muhammad Al-Khwarizmi regulatorika@yahoo.com

Mahruy Saidalieva Dr, Head of dept. "Regulatorika" Science and innovation center for information and communication technologies at the Tashkent University of Information Technologies named after Muhammad Al-Khwarizmi regulatorika@yahoo.com

\title{
REGULATORIKA OF THE IMMUNE SYSTEM AT THE CELLULAR LEVEL AT THE NORM AND TUMOR PROCESS
}

Abstract: This paper considers the method for modeling regulatorika of the immune system taking into account the numbers of circulating micro-RNA and CD4, CD8 lymphocytes concentration. Studies results have shown that in the model of the immune system there are the following regimes: extinction (apoptosis), stable oscillatory behavior (norm), irregular functioning (malignant neoplasm), and the effect of sharp destructive changes (metastasis).

Key words: modeling, regulatorika, functional-differential equations with delay, miRNA, cancer.

Language: Russian

Citation: Hidirova M, Saidalieva M (2017) REGULATORIKA OF THE IMMUNE SYSTEM AT THE CELLULAR LEVEL AT THE NORM AND TUMOR PROCESS. ISJ Theoretical \& Applied Science, 09 (53): 113-118.
Soi: http://s-o-i.org/1.1/TAS-09-53-17
Doi: crossef https://dx.doi.org/10.15863/TAS.2017.09.53.17

\section{УДК 576.35:517.948}

\section{РЕГУЛЯТОРИКА ИММУННОЙ СИСТЕМЫ НА КЛЕТОЧНОМ УРОВНЕ В НОРМЕ И ПРИ ОПУХОЛЕВОМ ПРОЦЕССЕ}

Аннотация: В статье рассматривается метод моделирования регуляторики иммунной системь с учетом численностей изиркулирующей микро-РНК, CD4, CD8 лимфоцитов. Результать исследований показывают наличие в модели регуляторики иммунной системы режимов угасания (апоптоз), стабильного автоколебательного поведения (норма), нерегулярного функционирования (злокачественным новообразованием) и эффекта резких деструктивных изменений (метастазой).

Ключевые слова: моделирование, регуляторика, функционально-дифференцииальнье уравнения с запаздыванием, микроРНК, рак.

\section{Introduction}

Бурное развитие компьютеризации и информатизации, происходящее в настоящее время, открывает широкие возможности внедрения достижений новых информационных технологий в научных исследованиях, разработки и применения средств вычислительных экспериментов. Последнее особо актуально в области медико-биологических научных исследований. Результаты анализа литературных данных показывают, что функционирование иммунной системы человека играет важную роль в формировании опухолевых процессов в организме [1-8]. Активация генов тесно связана с функционированием иммунной системы. Циркулирующие в крови некодирующие РНК являются регуляторами всех ключевых жизненно важных процессов. В последние годы наблюдается бурный рост работ по генной регуляции, связанный с открытием в геноме человека генов, кодирующих регуляторные молекулы - микроРНК, которые негативно регулируют экспрессию многих генов. МикроРНК синтезируется из более длинных предшественников и не кодирует белки. В большинстве случаев микроРНК действуют как репрессоры трансляции за счет связывания с иРНК. Аналогично оперонам прокариот или 
транскрипционным факторам эукариот, которые регулируют общий набор клеточных генов, одиночная miRNA обладает потенциалом регулировать множественные функционально родственные мРНК посттранскрипционно в ответ на стресс. Так, в ответ на повреждения ДНК или онкогенный стресс белок опухолевого супрессора p53 активирует транскрипцию miR-34a, которая в свою очередь регулирует экспрессию программы клеточного цикла и генов, отвечающих на повреждения ДНК, чтобы предупредить неадекватную клеточную пролиферацию. МикроРНК приводят к разрушению мРНК или подавлению трансляции с таргетной мРНК. При этом они способны подавлять трансляцию посредством различных механизмов: как на этапе инициации, так и в процессе элонгации. Механизм прекращения трансляции также зависит от степени комплементарности связывающихся областей микроРНК и мРНК. Деградация мРНК включает такие процессы, как деаденилирование, декапирование и экзонуклеарное расщепление молекулы мРНК. Однако до конца механизм действия микроРНК еще не изучен.

\section{Materials and Methods}

Как известно, в предотвращении опухолевых патологий активное участие принимают клеточные сообщества иммунной системы. Познание механизмов поддержания численности клеток иммунной системы (в частности, СД4, CD8 лимфоцитов) на нормальном уровне и анализ возможных путей нарушения данного механизма при канцерогенезе является одной из актуальных задач борьбы с раковыми заболеваниями. Координация структурно-функциональной организации огромного числа клеток в иммунной системе организма достигается посредством регуляторных механизмов. Важными звеньями данных регуляторных механизмов является система пролиферации и своевременное удаление клеток - апоптоз. Интуитивное понимание работы этих регуляторных механизмов в процессе регулирования активности многокомпонентной системы клеток, связанных посредством сложного взаимодействия механизмов положительных и отрицательных цепей обратной связи, является очень трудным. Здесь необходимы формальные математические методы и компьютерные инструментальные средства для моделирования и имитации соответствующих регуляторных механизмов. Клетки многоклеточного организма в ходе выполнения общих функций объединены в структурно-функциональные образования, состоящие из характерных групп клеток, выполняющих функции обновления, специализации, обмена веществ с окружающей средой, выполнения специфических функций и старения, т.е. объединены в функциональные единицы клеточных сообществ (ФЕКС), пространственное и функциональное образование из которых и составляет органы и ткани многоклеточного организма. Определение: связное множество (по пространству и (или) по времени) клеток называется ФЕКС, если в нем имеются делящиеся (М), растущие $\left(\mathrm{B}_{1}\right)$, дифференцирующиеся (D), выполняющие специфические функции $\left(\mathrm{S}_{1}, \ldots, \mathrm{S}_{\mathrm{n}}\right)$ и стареющие клетки $\left(\mathrm{B}_{2}\right)$, функционирующие взаимосвязанно, как единое целое ( $n$ - количество специфических функций ФЕКС) [9, 10]. На рис. 1 приведена схема ФЕКС для $n=2$. Предполагая для простоты наличие двух специфических функций $\begin{array}{llllll}\mathrm{S}_{1} & \text { и } & \mathrm{S}_{2} & \text { (функционирование } \mathrm{CD} 4, \mathrm{CD} 8\end{array}$ лимфоцитов), рассмотрим один из возможных вариантов исследования механизмов регуляции численности клеток в отдельных группах $\left(\mathrm{M}, \mathrm{B}_{1}\right.$, $\left.\mathrm{D}, \quad \mathrm{S}_{1}, \quad \mathrm{~S}_{2}, \quad \mathrm{~B}_{2}\right) \quad$ ФЕКС $\mathrm{c}$ помощью дифференциальных уравнений.

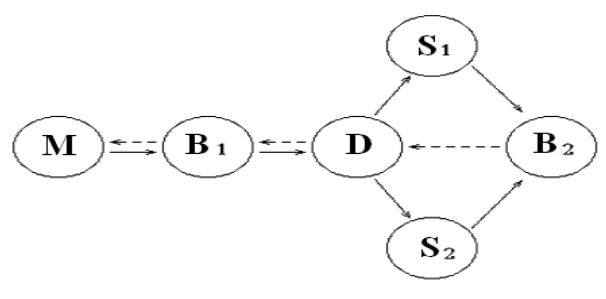

\section{Рисунок 1 - Схема ФЕКС (стрелки указывают переходы клеток из одной клеточной группы в другую, заштрихованные стрелки - обратные переходы).}

\begin{abstract}
Пусть характеризующие $X_{i}(t) \quad(i=1, \ldots, 6)$ - величины, численности делящихся, растущих, дифференцирующихся, выполняющих специфические функции и стареющих клеток в момент времени $t$. Составим уравнения для
\end{abstract}

\section{количественного}

описания

изменения численностей клеток в конкретных группах ФЕКС. Наиболее важной в функциональном смысле группой ФЕКС является группа размножающихся клеток путем деления. 
Скорость размножения зависит от количества потенциально способных к делению клеток, от веществ, способствующих делению (эффекторов), и от питательных веществ. Если предположить, что в ходе эволюции образовались ФЕКС с полезными для организма функциями, то проще всего принять, что количество эффекторов и поступающих в группу деления питательных веществ зависит от степени выполнения клеточным сообществом специфических функций, т.е. зависит от количества клеток в $S_{1}$ и $S_{2}$ группах. C учетом эффекта «давления среды» (торможения конечным продуктом) и возможности обмена клетками группы М с группой $\mathrm{B}_{1}$ можно написать, на основе метода моделирования регуляторики живых систем [11], следующее уравнение

$$
\begin{aligned}
& \frac{d X_{1}(t)}{d t}=a_{1}\left(\prod_{k}^{1,4,5} X_{k}\left(t-\tau_{k}\right)\right) e^{-\sum_{j=1}^{6} \delta_{j} X_{j}\left(t-\tau_{6}\right)}+ \\
& +b_{1} X_{2}\left(t-\tau_{1}\right)-a_{2} X_{1}(t)
\end{aligned}
$$

где $a_{1}-$ постоянная скорости размножения; $b_{1}, a_{2}-$ постоянные скорости обмена клеток группы М с группой $\mathrm{B}_{1} ; \quad \delta_{i}-$ коэффициент, характеризующий давление среды; $\tau_{i}-$ время перехода $(i=1,2, \ldots, 6)$. С учетом переходов клеток из одной группы ФЕКС в другую, можно написать следующую систему линейных функционально-дифференциальных уравнений для изменения численностей клеток в группах $\mathrm{B}_{1}$, $\mathrm{D}, \mathrm{S}_{1}, \mathrm{~S}_{2}$ и $\mathrm{B}_{2}$ :

$\frac{d X_{2}(t)}{d t}=a_{2} X_{1}\left(t-\tau_{1}\right)+b_{2} X_{3}\left(t-\tau_{2}\right)-\left(b_{1}+a_{3}\right) X_{2}(t)$

$\frac{d X_{3}(t)}{d t}=a_{3} X_{2}\left(t-\tau_{2}\right)+b_{3} X_{6}\left(t-\tau_{6}\right)-\left(b_{2}+a_{4}+a_{5}\right) X_{3}(t) ;$

$\frac{d X_{4}(t)}{d t}=a_{4} X_{3}\left(t-\tau_{3}\right)-a_{6} X_{4}(t) ;$

$\frac{d X_{5}(t)}{d t}=a_{5} X_{3}\left(t-\tau_{3}\right)-a_{6} X_{5}(t)$

$\frac{d X_{6}(t)}{d t}=a_{6}\left(X_{4}\left(t-\tau_{5}\right)+X_{5}\left(t-\tau_{5}\right)\right)-\left(a_{6}+a_{7}\right) X_{6}(t)$.

Уравнения (1) и (2) составляют замкнутую систему функционально-дифференциальных уравнений динамики численности клеток ФЕКС. Теоремы существования и единственности непрерывных решений, а также приближенные решения данных уравнений на РС могут быть получены с использованием метода последовательного интегрирования БеллманаКука при задании начальной функции на отрезке длины $\tau=\max _{i}\left(\tau_{i}\right) ; \quad i=1,2, \ldots, 6 . \quad$ Система уравнений (1)-(2) носит сложный характер и применение ее для качественного и количественного анализа механизмов функционирования клеток иммунной системы требует разработки их модельных систем. Модельные системы, сохраняя основные свойства исходных уравнений, являются относительно простыми системами уравнений с минимально возможным числом соотношений и параметров. Это позволяет проводить, в большинстве случаев, успешный аналитический анализ характерных решений, а также определять основные режимы поведения рассматриваемых математических моделей. Для анализа регуляторики динамики численности CD4 и CD8 лимфоцитов мы можем упростить систему (1)(2). Нас интересуют наиболее общие механизмы поддержания гомеостазиса CD4 и CD8 лимфоцитов в течение жизни организма. Для анализа динамики численности CD4 и CD8 лимфоцитов с учетом действия на их активности циркулирующей микро-РНК, мы можем использовать следующую систему функционально-дифференциальных уравнений с запаздыванием, разработанной на основе метода регуляторики живых систем [11]

$$
\begin{aligned}
& \frac{d X_{1}(t)}{d t}=a_{1} R(t-h) e^{-d_{1} K(t-h)}-b_{1} X_{1}(t) \\
& \frac{d X_{2}(t)}{d t}=a_{2} X_{1}(t-h)-b_{2} X_{2}(t) \\
& \frac{d X_{3}(t)}{d t}=a_{3} X_{2}(t-h)-b_{3} X_{3}(t)
\end{aligned}
$$

где

$$
\begin{gathered}
R(t-h)=X_{1}(t-h) X_{2}(t-h) X_{3}(t-h) ; \\
K(t-h)=X_{1}(t-h)+X_{2}(t-h)+X_{3}(t-h)
\end{gathered}
$$

с начальными условиями

$$
\begin{gathered}
X_{i}(t)=\varphi_{i}(t) \text { при } \quad t_{0}-h \leq t \leq t_{0}\left(t_{0}>h\right) ; \\
i,=1,2,3 .
\end{gathered}
$$

$X_{l}(t)$ - безразмерная величина, выражающая численность циркулирующей микро-РНК; $X_{2}(t)-$ безразмерная величина, выражающая численность CD4 лимфоцитов; $X_{3}(t)$ - безразмерная величина, выражающая численность CD8 лимфоцитов; $a_{1}$, $a_{2}, a_{3}$ - выражают скорости воспроизводства микро-РНК, CD4 и CD8 лимфоцитов соответственно; $b_{1}, b_{2}, b_{3}$ - выражают скорость гибели микро-РНК, CD4 и CD8 лимфоцитов, соответственно.

Дифференциально-разностные уравнения (3) относятся к классу функциональнодифференциальных уравнений и обладают бесконечным числом базисных функций. Задача 
Коши для данных уравнений (так называемая «начальная задача») формулируется следующим образом. Пусть заданы начальные функции $\varphi_{1}(t), \varphi_{2}(t), \varphi_{3}(t)$ - непрерывные на $[-h, 0]$ и

$$
\begin{gathered}
X_{i}(t)=\varphi_{i}(t) \quad \text { при } t \in[-h, 0], \\
i=1,2,3 .
\end{gathered}
$$

Требуется определить непрерывное решение (3) при $t>0$, удовлетворяющее (4). Рассмотрим отрезок $[0, h]$. Используя (4), можно записать (3) в виде:

$$
\begin{aligned}
& \frac{d X_{1}(t)}{d t}=a_{1} R \varphi(t-h) e^{-d_{1} K \varphi(t-h)}-b_{1} X_{1}(t) \\
& \frac{d X_{2}(t)}{d t}=a_{2} \varphi_{1}(t-h)-b_{2} X_{2}(t) \\
& \frac{d X_{3}(t)}{d t}=a_{3} \phi_{1}(t-h)-b_{3} X_{3}(t),
\end{aligned}
$$

где

$$
\begin{gathered}
R \varphi(t-h)=\varphi_{1}(t-h) \varphi_{2}(t-h) \varphi_{3}(t-h) \\
K \varphi(t-h)=\varphi_{1}(t-h)+\varphi_{2}(t-h)+\varphi_{3}(t-h) .
\end{gathered}
$$

Путем введения замены переменных

$$
X_{i}(t)=Y_{i}(t) e^{-b_{i} t}, \quad i=1,2,3
$$

уравнения (5) можно привести к следующему виду

$$
\begin{gathered}
\frac{d Y_{1}(t)}{d t}=a_{1} e^{b_{1} t} R \varphi(t-h) e^{-d_{1} K \varphi(t-h)} \\
\frac{d Y_{2}(t)}{d t}=e^{b_{2} t} a_{2} \varphi_{1}(t-h) ; \\
\frac{d Y_{3}(t)}{d t}=e^{b_{3} t} a_{3} \varphi_{1}(t-h)
\end{gathered}
$$

Отсюда, для $t \sqsubset(0, h]$, получаем

$$
\begin{gathered}
Y_{1}=\varphi_{1}(0)+\int_{0}^{t} a_{1} e^{b_{1} s} R \varphi(\mathrm{s}-h) e^{-d_{1} K \varphi(\mathrm{s}-h)} d s \\
Y_{2}(t)=\varphi_{2}(0)+\int_{0}^{t} e^{b_{2} s} a_{2} \varphi_{1}(s-h) d s \\
Y_{3}(t)=\varphi_{3}(0)+\int_{0}^{t} e^{b_{3} s} a_{3} \varphi_{1}(s-h) d s
\end{gathered}
$$

Следовательно,

$$
\begin{aligned}
& X_{1}=e^{-b_{1} t}\left(\varphi_{1}(0)+\int_{0}^{t} a_{1} e^{b_{1} s} R \varphi(\mathrm{s}-h) e^{-d_{1} K \varphi(\mathrm{s}-h)} d s\right) \\
& X_{2}(t)=e^{-b_{2} t}\left(\varphi_{2}(0)+\int_{0}^{t} e^{b_{2} s} a_{2} \varphi_{1}(s-h) d s\right)
\end{aligned}
$$

$$
X_{3}(t)=e^{-b_{3} t}\left(\phi_{3}(0)+\int_{0}^{t} e^{b_{3} s} a_{3} \phi_{1}(s-h) d s\right) \text {. }
$$

Принимая полученные решения за начальные функции и повторяя процедуру интегрирования, получаем решения на $[h, 2 h]$ и т.д. Такое последовательное интегрирование позволяет получить непрерывное решение при $t>0$. Непрерывность следует из непрерывности начальных функций и характера построения решения. При реализации (1) на современных РС возникает задача построения решений по заданным дискретным значениям искомых переменных, которая является актуальной и при количественном описании биологических процессов функционально-дифференциальными уравнениями запаздывающего типа при наличии только дискретных экспериментальных данных. В зависимости от способа задания начальных данных могут быть применены различные способы последовательного интегрирования функционально-дифференциальных уравнений запаздывающего типа. Если начальные данные заданы внутри отрезка длины $h$ и их количество достаточно, чтобы характеризовать поведение системы на начальном отрезке, то можно применять метод последовательного интегрирования. Иногда начальные данные могут быть заданы не внутри отрезка длины $h$, а разбросаны на отрезке, намного превышающем длину $h$. Тогда мы можем воспользоваться построением приближенных решений в окрестности требуемой точки путем линеаризации рассматриваемых уравнений, так как для определенного класса линейных функционально-дифференциальных уравнений запаздывающего типа данная задача является разрешимой и соответствующие результаты можно использовать при оценке поведения решений (1) в рассматриваемой области фазового пространства.

Также анализ решений системы (1) осуществляется методами качественного исследования функциональнодифференциальных уравнений. Определение критических точек позволяет установить «истоки» и «стоки» потоков решений, т.е. аттракторов системы. В зависимости от определения нахождения системы в сфере влияния конкретного аттрактора можно выяснить характер поведения решений. Например, если система находится в сфере влияния тривиального аттрактора, то решения, в конечном итоге, будут стремиться к нулю. Наличие колебательных решений определяется нарушением устойчивости положительного аттрактора: в этом случае происходит бифуркация решений и вокруг положительного аттрактора появляются (согласно теореме 
Пуанкаре-Бендиксона) автоколебательные решения - предельные циклы типа Пуанкаре. Во многих случаях полезно строить соответствующие редуцированные уравнения (так называемые «модельные системы») на основе биологических, биофизических соображений и математических приемов. Это позволяет эффективно применять компьютерные способы анализа общих закономерностей поведения решений. Предварительно анализируя области однородного поведения в параметрическом пространстве, осуществляется их исследование в фазовом пространстве с помощью теории качественного анализа функционально-дифференциальных уравнений и разработанных специальных программ. Следует особо отметить эффективность модельных систем в виде дискретных рекуррентных уравнений. Анализ деформации фазового пространства в окрестности выбранной точки позволяет определять характер регулярных, бифуркационных, нерегулярных и деструктивных поведений иммунной системы при раковых новообразованиях, что позволяет исследовать взаимосвязи частоты хромосомных аберраций $c$ полиморфными вариантами генов. Для оценки: устойчивости решений, существования колебательных решений, возникновения странного аттрактора и нерегулярных колебаний могут быть использованы методы бифуркационного и фрактального анализа, а также методы обнаружения эффекта «черная дыра» - срыва решений к тривиальному аттрактору. Следует отметить особую важность определения механизмов возникновения эффекта «черная дыра» - области деструктивных изменений. Область деструктивных изменений можно идентифицировать как начало развития рака с летальным исходом. Анализ характерных поведений решений уравнений (3) с использованием математической и компьютерной моделей регуляторики организма, методов качественного исследования функциональнодифференциальных уравнений показывает наличие в модели регуляторики иммунной системы режимов угасания (А), устойчивого стационарного состояния (В), стабильного автоколебательного поведения (C), нерегулярного функционирования (D) и эффекта резких деструктивных изменений (Е) - эффекта «черная дыра». В области хаоса могут наблюдаться случаи малых r-windows, внутри которых поведение клеток иммунной системы носит нормальный характер. Нерегулярное поведение и «черная дыра» могут быть идентифицированы неконтролируемым размножением клеток (злокачественным новообразованием) и резким деструктивным изменением регуляторики клеточных сообществ иммунной системы (метастазой). Область угасания можно идентифицировать с областью апоптоза. Во время онкогенеза экспрессия регулирующих апоптоз генов, может существенно изменяться.

\section{Conclusion}

Таким образом, особенно актуально исследование структурных особенностей области регулярного поведения, степени хаотичности и области резких деструктивных изменений вследствие актуальности ранней диагностики раковых заболеваний. Область нерегулярных колебаний характеризуется нарушением системы регуляции иммунной системы с последовательным ухудшением функциональной активности, возникновением спонтанных хромосомных аберраций и патологических аллелей. Различная степень хаотичности области динамического хаоса может идентифицироваться различными стадиями ракового заболевания. Степень «хаотичности» может быть оценена по значению энтропии Колмогорова.

\section{References:}

1. Sheller B, D'Alessandro D. (2015) Analysis of a cancer dormancy model and control of immuno-therapy. Math Biosci Eng. 2015 Oct 1;12(5):1037-53.

2. Yang J, Martcheva M, Wang L. (2015) Global threshold dynamics of an SIVS model with waning vaccine-induced immunity and nonlinear incidence. Math Biosci. 2015 Oct;268:1-8.
3. Mazzocco P, Bernard S, Pujo-Menjouet L. (2017) Estimates and impact of lymphocyte division parameters from CFSE data using mathematical modelling. PLoS One. 2017

4. Luzyanina T, Cupovic J, Ludewig B, Bocharov G. (2013) Mathematical models for CFSE labelled lymphocyte dynamics: asymmetry and time-lag in division. J Math Biol. 2013; p. 1547-1583. 


\begin{tabular}{|c|c|c|c|c|c|c|}
\hline Impact Factor: & $\begin{array}{l}\text { ISRA (India) } \\
\text { ISI (Dubai, UAE } \\
\text { GIF (Australia) } \\
\text { JIF }\end{array}$ & $\begin{array}{l}=1.344 \\
=0.829 \\
=0.564 \\
=1.500\end{array}$ & $\begin{array}{l}\text { SIS (USA) } \\
\text { PИНЦ (Russia) } \\
\text { ESJI (KZ) } \\
\text { SJIF (Morocco) }\end{array}$ & $\begin{array}{l}=0.912 \\
=0.207 \\
=\mathbf{3 . 8 6 0} \\
=\mathbf{2 . 0 3 1}\end{array}$ & $\begin{array}{l}\text { ICV (Poland) } \\
\text { PIF (India) } \\
\text { IBI (India) }\end{array}$ & $\begin{array}{l}=6.630 \\
=1.940 \\
=4.260\end{array}$ \\
\hline
\end{tabular}

5. Dowling M.R, Kan A, Heinzel S, Zhou J.H, Marchingo J.M, Wellard C.J, et al. (2014) Stretched cell cycle model for proliferating lymphocytes. Proceedings of the National Academy of Sciences. 2014;111(17):6377-6382.

6. R. Antia, S. Pilyugin, R. Ahmed. (1998) Models of immune memory: on the role of cross-reactive stimulation, competition, and homeostasis in maintaining immune memory. Proc. Natl. Acad. Sci. USA, 95 (1998), no. 25, 14926-14931.

7. R.P. Araujo, D.L.S. McElwain. (2004) A history of the study of solid tumor growth: the contribution of mathematical modeling.Bull. Math. Biol., 66 (2004), 1039-1091.

8. L.G. de Pillis, A.E. Radunskaya, C.L. Wiseman. (2005) A validated mathematical model of cell-mediated immune response to tumor growth. Cancer. Res., 65 (2005), no. 17, 7950-7958.

9. Saidalieva M. (2003) Modelling of Regulation Mechanisms of Cellular Communities //Scientiae Mathematicae Japonicae. Published bimonthly by International Society for Mathematical Sciences, Kyoto University Japan, Vol. 58.No 2. 2003. P. 415-421.

10. Saidalieva M. (2008) Mathematical and Computer Modelling Regulatorika of Organisms Cellular Communities at Anomalies //Scientiae Mathematicae Japonicae, 2008. Japan, Published bimonthly by International Society for Mathematical Sciences, Kyoto University, Vol. 67. No 2. P. 161-171.

11. Hidirov B.N. (2014) Selected works on mathematical modeling living systems regulatorika. Moscow-Ijevsk, 2014, 304 P. (in Russian). 